\title{
Conteo automático de cubos en evaluación de destreza manual usando sensores de proximidad
}

\author{
Víctor Pozo, Edwin Daniel Oña*, Alberto Jardón, Carlos Balaguer \\ Robotics Lab, Universidad Carlos III de Madrid, Avda. Universidad 30, Leganés (Madrid), España, \\ 100303638@alumnos.uc3m.es, \{*eona, ajardon, balaguer\}@ing.uc3m.es
}

\begin{abstract}
Resumen
En este artículo, se presenta el desarrollo de un sistema basado en sensores de proximidad que permite obtener de forma automática la puntuación del Box and Blocks Test (BBT), destinado a medir la destreza manual gruesa. La evaluación del BBT se basa en el máximo número de cubos desplazados en una ventana de tiempo. Por ese motivo, se han utilizado varios sensores de proximidad basados en detección infrarroja, para sensorizar la trayectoria de desplazamiento de los cubos. En primer lugar, se describe el método utilizado para la automatización de la puntuación del test, describiendo los sensores y el diseño del prototipo. A continuación se muestra la tasa de acierto obtenida en base a varios experimentos. Finalmente, se exponen las conclusiones y futuros trabajos. Los resultados presentados apoyan el uso de métodos automatizados en procesos de rehabilitación, que pueden generar resultados objetivos e información adicional sobre el desempeño del usuario.
\end{abstract}

Palabras clave: Evaluación, Automático, Extremidad superior, Destreza manual, Rehabilitación, Sensores proximidad.

\section{INTRODUCCIÓN}

En la actualidad, la gran demanda de los servicios sanitarios hace necesaria una atención médica rápida y de calidad. Aplicaciones y dispositivos electrónicos en entornos hospitalarios (e-Health) hacen posible mejorar la agilidad en procesos como la gestión de citas médicas, ensayos clínicos, pruebas para diagnóstico o rehabilitación [11].

Haciendo referencia a los procesos de rehabilitación tras un accidente cerebrovascular (ACV), se puede observar que muchos son procesos de larga duración en los que los resultados visibles se aprecian tras un gran número de sesiones, que en ciertos casos abarcan periodos de varios años. Por este motivo, es necesario tener un seguimiento efectivo de los resultados obtenidos durante la rehabilitación, ya que evaluar su efectividad terapéutica es particularmente importante [8].
Para realizar dicha evaluación, los terapeutas utilizan escalas estandarizadas diseñadas para medir disfuncionalidad a diferentes niveles. El FuglMeyer Assessment (FMA) test es uno de los más utilizados en ensayos clínicos, permitiendo valorar función motora, balance, sensación y rangos de movimiento de las extremidades superiores e inferiores. Centrándose en valoración de la función motora de extremidades superiores, tenemos el Wolf Motor Function Test (WMFT) o el Action Research Arm Test (ARAT) que permiten valorar la autonomía para el desempeño de tareas. En el caso de evaluar la destreza manual se utiliza habitualmente el Box and Blocks Test (BBT).

Estos ejercicios son susceptibles de ser automatizados, proporcionando una evaluación más objetiva de la efectividad del tratamiento. Actualmente, los robots se consideran herramientas de terapia avanzadas bajo la guía del terapeuta, principalmente enfocados en la rehabilitación como intervención [6]. Sin embargo, la implementación de sistemas automáticos de evaluación puede ayudar a conseguir procesos de rehabilitación más autónomos y objetivos.

\subsection{TRABAJOS RELACIONADOS}

Se pueden encontrar trabajos de investigación enfocados en automatizar escalas de evaluación funcional como las mencionadas anteriormente.Por ejemplo, en [7] se propone un método automático basado en el FMA, usando sensores de bajo coste que registran datos de los movimientos del usuario, obteniendo resultados similares. En [10] se presenta otro intento de automatizar parte del FMA, en este caso, utilizando acelerómetros y centrándose en los movimientos de hombro y codo.

Una evaluación automática basada en el WMFT se plantea en [9]. Utilizando sensores vestibles, se estima el tiempo que se tarda en completar 7 de las 17 tareas del WMFT. Un estudio enfocado en la automatización el ARAT se presenta en [2]. En dicho trabajo, se ha planteado la automatización de la sección 4 del ARAT, por medio de la sensorización de uno de los objetos utilizados en la tarea, en este caso un cubo de $7.5 \mathrm{~cm}$. 
Por otro lado, en [1] se presenta una versión automática del BBT. En este trabajo, utilizando un sensor Kinect, se implementa un algoritmo para contar los cubos desplazados con un índice de acierto del $100 \%$ hasta 20 cubos. Además, detecta la mano y sus movimientos. Un estudio más reciente, y utilizando la segunda versión del sensor Kinect y visión por computador, es presentado en [5]. En este caso, se logra aumentar el porcentaje de acierto en el conteo de cubos del $100 \%$ hasta 30 cubos. Además, dicho estudio desarrolla una interfaz gráfica que permite abordar la administración automática de la prueba.

Se puede observar que un objetivo común es obtener plataformas de evaluación automática que sean objetivas, que tengan repetitividad, pudiendo aportar información adicional a la que se obtendría con la escala tradicional.

\section{MATERIAL Y MÉTODO}

En este artículo se ha seleccionado como caso de estudio el Box and Blocks Test (BBT), diseñado para evaluar la destreza manual gruesa. En la Figura 1 se muestra la dinámica en el desarrollo del BBT. Al inicio de la prueba, todos los cubos se encuentran en uno de los compartimentos, quedando el otro vacío. El usuario debe desplazar tantos cubos como pueda al compartimento vacío, superando la barrera central.

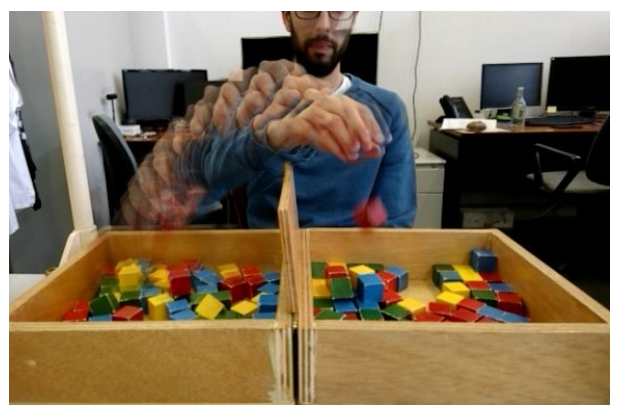

Figura 1: Usario realizando el BBT.

Analizando el desarrollo de la prueba, se puede observar que la dinámica del test se mantiene, tanto si se ejecuta con la mano derecha o izquierda. Para automatizar la puntuación del BBT, será necesario registrar la caída de los cubos al compartimento vacío, durante el periodo de tiempo definido por la prueba.

Así, el objetivo principal de este trabajo es implementar un método, basado en sensores de proximidad, que permita obtener de forma automática la puntuación del BBT. Además, el sistema implementado debe ser sencillo, de bajo coste y con el mínimo número de sensores.

\subsection{EL BOX \& BLOCKS TEST (BBT)}

El BBT es un sistema de medida individual de la destreza manual gruesa y coordinación, clínicamente validado. El test está formado por una caja de madera con dos compartimentos cuadrados de $290 \mathrm{~mm}$ de lado, y 150 cubos de madera de $25 \mathrm{~mm}$ (Figura 2). Entre los dos compartimentos se ubica una barrera de $100 \mathrm{~mm}$ de alto.

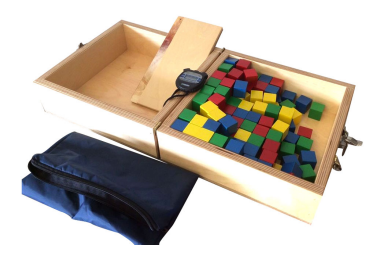

Figura 2: Box and Blocks Test (BBT).

El objetivo del test es desplazar la mayor cantidad posible de cubos de un lado de la caja al otro en un minuto (Figura 1). Para la puntuación, se cuenta manualmente el total de cubos desplazados. El desarrollo del test se comprende de tres etapas: un periodo de entrenamiento de 15 segundos, ejecución del test con la mano dominante o no afectada durante un minuto, y ejecución del test con la mano no dominante o afectada durante un minuto. Para que el desplazamiento del cubo sea válido, la mano del sujeto debe sobrepasar la barrera central de la caja. Los cubos lanzados desde una parte de la caja a la otra no son válidos. Además, el desplazamiento de cubos se debe realizar de uno en uno. En caso de desplazar más de un cubo a la vez, se contarán como uno solo. Para realizar la prueba, se ubica el BBT sobre una mesa y el usuario sentado delante del test realiza el ejercicio. El evaluador, lee las instrucciones del test al paciente antes de iniciar la evaluación. Las reglas completas y las instrucciones del test, se pueden consultar en [3].

\subsection{SENSORES DE PROXIMIDAD}

Para esta aplicación se ha utilizado el sensor de proximidad SI1143 [4]. Dicho sensor es un detector de proximidad fotoeléctrico basado en el efecto de reflexión óptica infrarroja difusa que permite detectar, tanto objetos como la intensidad de luz ambiente.

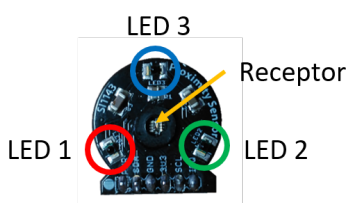

Figura 3: Sensor de proximidad SI1143 
Este sensor, mostrado en la Figura 3, cuenta con tres diodos LED emisores de infrarrojos y un dispositivo receptor. Los LED's emisores se encuentran dispuestos en forma triangular, ocupando los vértices de un triángulo equilátero, mientras que en el centro del triángulo se encuentra el elemento receptor. El valor de salida que ofrece el sensor viene dado por un conversor A/D (ADC).

En condiciones óptimas, el sensor puede detectar un objeto a distancias de hasta $50 \mathrm{~cm}$. A la hora de detectar objetos en movimiento, su rango de detección se ve reducido hasta los $30 \mathrm{~cm}$ de distancia máxima. En objetos muy pequeños la cantidad de luz reflejada se reduce hasta en una cuarta parte, por lo que a distancias superiores a $20 \mathrm{~cm}$ aumenta la dificultad para detectar dicho objeto.

\subsection{1. Área de detección}

Para modelar el área de detección, se puede tomar como forma geométrica de estudio un cono, en el que el vértice se corresponde al diodo y la base a la superficie de máxima detección. Las dimensiones de las superficies de detección han sido obtenidas experimentalmente.

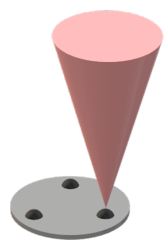

(a)
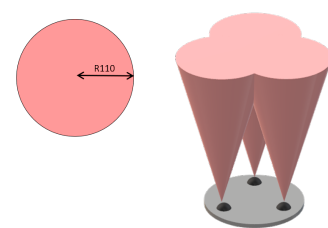

(b)

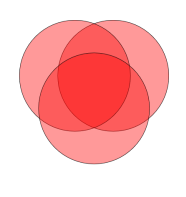

Figura 4: Área de detección implementando los tres emisores LED del sensor SI1143.

A la hora de utilizar un único LED (Figura 4-a), se obtiene un radio de detección máximo de $110 \mathrm{~mm}$. Este radio varía entre $110 \mathrm{~mm}$ y $90 \mathrm{~mm}$ a lo largo de las distancias que se encuentran en el rango entre $100 \mathrm{~mm}$ y $250 \mathrm{~mm}$. Para distancias inferiores, el radio de detección se reduce considerablemente siguiendo una curva exponencial, generando un ángulo muerto. Para aumentar la capacidad de detección del sensor se pueden utilizar los tres LED emisores de los que dispone (Figura 4-b), incrementando el radio de detección hasta $130 \mathrm{~mm}$. De esta manera, se genera una zona central con mayor potencia lumínica, por lo que los objetos son más fáciles de detectar.

\subsubsection{Detección de objetos}

Por un lado, el caso más sencillo para la detección de un objeto es al triangular su posición utilizando dos de los LEDs. De este modo se puede conocer si el objeto se encuentra a la izquierda o a la derecha del sensor, además de la distancia a la que se encuentra. Suponiendo que un objeto se encuentra en el campo de detección del sensor, este reflejará la luz emitida por los dos LED al dispositivo receptor. Trazando una circunferencia con centro en cada LED de radio igual a el valor detectado (Figura 5), se puede conocer la posición del objeto mediante la intersección de ambas circunferencias. Para generar el seguimiento de la trayectoria del objeto basta con relacionar su posición con el instante de tiempo en el que se detecta.

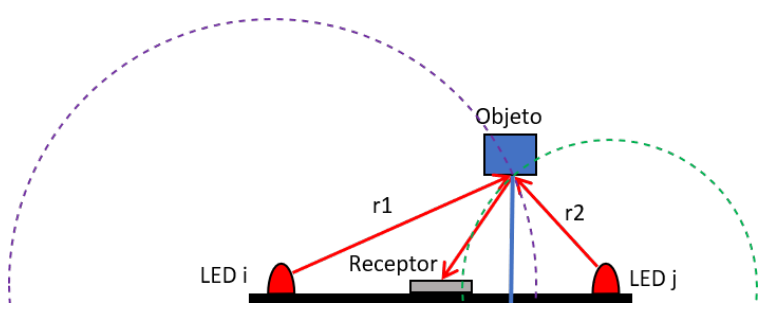

Figura 5: Triangulación básica de objeto.

Por otro lado, al disponer de tres fuentes emisoras se puede analizar el desplazamiento de un objeto en función de los flancos de entrada en el área de detección del sensor. Al desplazarse un objeto por encima del sensor hará que uno de los LED emisores sea el primero en detectar, considerando esta zona el flanco de entrada (ver Figura 6). Del mismo modo, un LED será el último en dejar de detectar, siendo este el flanco de salida.

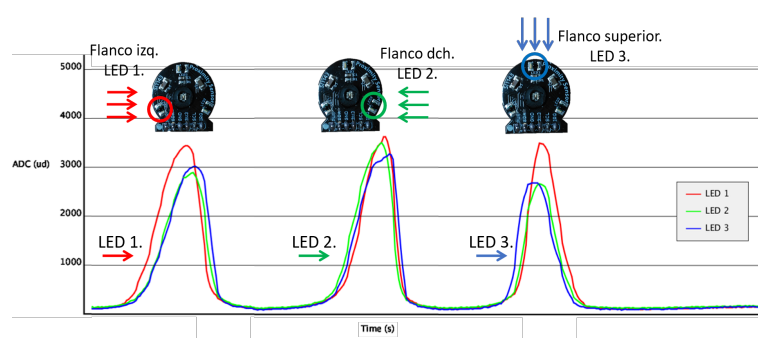

Figura 6: Flancos de entrada por cada LED.

Aplicando este método se pueden conocer la zona de entrada y salida de un objeto sin necesidad de triangular su posición. Por el contrario, no se pueden determinar los movimientos que se realicen dentro de la zona de detección. Además, el número de trayectorias es reducido ya que solo se presentan tres flancos claros. En cambio, para funciones de reconocimiento de movimientos de objetos mayores, como los de una mano, se pueden implementar aplicaciones fiables.

\section{SISTEMA AUTOMÁTICO DE CONTEO DE CUBOS}

La estructura de la caja del BBT permite disponer los sensores de proximidad en distintas zonas, 
diferenciando entre los laterales del compartimento y la pieza divisoria. Tras estudiar el funcionamiento del sensor y atendiendo a los resultados experimentales obtenidos en la instalación de un solo sensor y una pareja de sensores en distintas posiciones y orientaciones en uno de los compartimentos, se obtienen las siguientes directrices que se han tenido en cuenta para el diseño final:

- La distancia máxima real de detección de los sensores es de $20 \mathrm{~cm}$, por lo que un solo sensor no es capaz de cubrir ni el ancho completo (26 $\mathrm{cm})$, ni las diagonales del compartimento.

- Los sensores que no están colocados en un mismo lateral producen interferencias entre ellos.

- Los sensores presentan un ángulo de visión muerto a su alrededor. Dos sensores colocados en un mismo lateral no son suficientes para cubrir dichos ángulos.

- Los sensores instalados en las esquinas del compartimento detectan las zonas de pared lateral mas cercanas, alterando la señal de detección.

- Al elevar la posición de los sensores hasta el exterior del compartimento se reducen las interferencias provocadas por un efecto similar a "crosstalk".

De esta manera, se ha definido que la mejor configuración para el sistema de detección es que los sensores estén colocados en un mismo lateral y en una posición elevada respecto del compartimento.

\subsection{SISTEMA IMPLEMENTADO}

Atendiendo a las consideraciones anteriores, se ha seleccionado la pieza divisoria del BBT para la ubicación de los sensores. Esta pieza se eleva 10 cm por encima del borde superior de los compartimentos, y además permite la colocación de los sensores sin alterar la estructura física del BBT y evita entorpecer el desarrollo del test. Se decide sensorizar solo uno de los laterales, puesto que esta pieza es móvil y se puede dar la vuelta para detectar los cubos en el otro compartimento.

Se utilizan tres sensores colocados en un lateral de la pieza divisoria a una cota de $8.5 \mathrm{~cm}$ y separados $7.5 \mathrm{~cm}$ entre sí (Figura 7-a). Con esta posición se obtiene una distancia de $16 \mathrm{~cm}$ respecto la base del compartimento (Figura 7-b), reduciendo las interferencias generadas por la presencia de distintos sensores ya que tanto la pared más alejada como la base se encuentran en una zona infrarroja de baja intensidad.

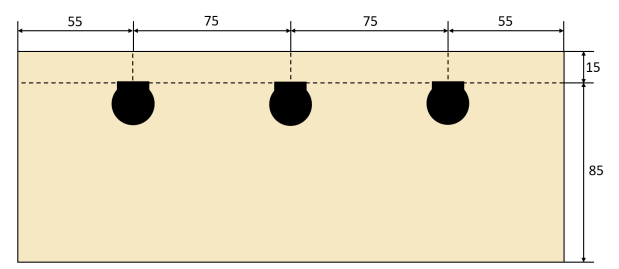

(a)

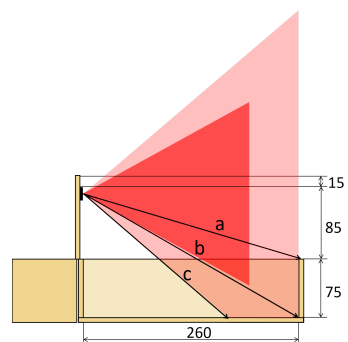

(b)

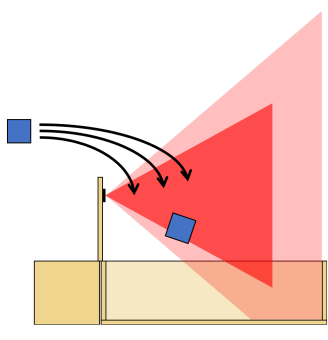

(c)
Figura 7: Sistema propuesto para conteo automático de cubos. (a) Disposición de los sensores SI1143 en la pieza divisoria del BBT; (b) Distancias máximas entre el sensor y el compartimento ( $\mathrm{a}=273.3 \mathrm{~mm}, \mathrm{~b}=305.3 \mathrm{~mm}, \mathrm{c}=226.3 \mathrm{~mm}$ ); (c) Trayectorias normales de recepción de cubos.

Como se puede ver en la Figura 7-b, las zonas más alejadas del sensor (distancias superiores a $20 \mathrm{~cm}$ ) quedan sin sensorizar o con una capacidad de detección muy baja. Teniendo en cuenta la velocidad de caída de los cubos, el sistema de sensores es incapaz de detectarlos en esta zona. En este caso, se va a asumir este error ya que en un desarrollo normal de la prueba BBT los cubos no deberían caer directamente por esa zona, sino que primero atravesarían una zona más próxima a los sensores (Figura 7-c).

Por otro lado, los tres sensores se han dispuesto de tal forma que los LED 1 y LED 2 de cada sensor queden alineados en la parte superior, mientras que el LED 3 queda en la parte inferior, como se muestra en la Figura 8. De esta manera, se forma una barrera superior en la que operan seis LED (dos LED por sensor) y una barrera inferior de apoyo con tres LED (un LED por sensor). Cuando un cubo se deja caer la barrera superior lo registra, en caso de no hacerlo la barrera inferior funciona como soporte de ayuda a la detección.

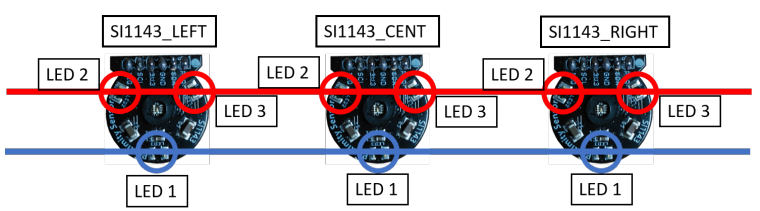

Figura 8: Orientación de los sensores generando una barrera de detección principal y otra de apoyo. 


\subsubsection{Gestión de ángulos muertos}

Utilizando tres sensores se consigue cubrir el área necesaria para detectar los cubos utilizados en el BBT, cubriendo las zonas de ángulo muerto.

En la gráfica de la Figura 9 se muestran, desde la vista de planta, los límites de detección en las zonas cercanas al sensor. Esta representa a los tres sensores a una distancia entre ellos de $7.5 \mathrm{~cm}$. La línea horizontal representa el tamaño de un cubo (2.5 cm de lado). Al mantener esta separación entre los sensores, se consigue evitar el fallo de registro de la caída de cubos por zonas de ángulo muerto. Debido al tamaño del cubo siempre podrá ser detectado, incluso si cae muy cerca de la propia pieza divisoria o en las esquinas del compartimento.

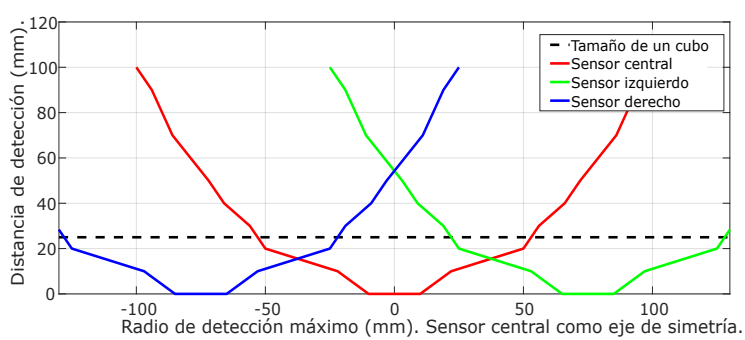

Figura 9: Límites de detección de tres sensores Si1143 instalados en la pieza divisoria del BBT.

\subsection{PROCESO DE CONTEO AUTOMÁTICO}

La detección de cubos automatizada mediante los sensores SI1143 se divide en tres fases: Reconocimiento del compartimento, Tratamiento de la señal, y Algoritmo de detección.

\subsubsection{Reconocimiento del compartimento}

Una vez iniciada la aplicación los sensores recopilan datos del entorno durante 0.2 segundos detectando los laterales del compartimento, así como posibles objetos cercanos como el propio cuerpo del paciente. Estos datos preparan una Supresión del Primer Plano ( Foreground Suppression" FGS), limitando el área de detección a la zona interior del compartimento de recepción de cubos. De este modo, todo aquello susceptible de ser detectado alrededor de la estructura del BBT durante la prueba será descartado.

El valor de la señal ADC promedio y el máximo durante el reconocimiento son la base del cálculo para el FGS. El valor promedio marca un primer valor umbral, al que se le suma un porcentaje de error obtenido mediante la relación del propio promedio y el valor máximo. Además, se aplica un factor de corrección que asegura un valor FGS final (ecuación (1)) que delimite la detección al interior del compartimento. En un entorno libre de objetos dicho factor de corrección toma un valor de 1.1 .

$$
A D C_{F G S}=A D C_{P m}(\text { factor }+ \text { Error })
$$

\subsubsection{Tratamiento de señal}

Durante el funcionamiento del sistema aparecen una serie de perturbaciones en la señal obtenida por los sensores.

Por un lado, se aprecia una tendencia constante en la lectura de los sensores, pero sus valores oscilan debido al ruido ambiente. En el caso de no detectar (vacío) se obtienen $375 \pm 15$ unidades ADC.

Por otro lado, al encontrarse tres sensores operando en un recinto semi-cerrado, los haces de luz infrarrojo de un sensor pueden afectar a los demás, apareciendo perturbaciones en sus señales. Estas son similares a la señal obtenida en la detección de cubos en las zonas centrales del BBT, lo que genera falsos positivos.

Para solventar estos problemas el sistema aplica un alisado exponencial (ecuación (2)) a la señal, utilizando un factor de alisado de 0.8. Además, se toma como referencia de detección el valor $\mathrm{ADC}_{\mathrm{FGS}}$ de la fase de reconocimiento. Estas dos medidas permiten analizar una señal más constante y suprime la mayoría de perturbaciones.

$$
A D C_{i}=A D C_{i-1}+\operatorname{cte}\left(A D C_{\text {real }}-A D C_{i-1}\right)
$$

\subsubsection{Algoritmo de detección}

En la Figura 10 se muestra el diagrama de flujo del proceso automatizado para contar cubos, acotándose el proceso a una duración de 60 segundos según la normativa del BBT.

Cuando un cubo es lanzado al interior del compartimento sigue una trayectoria recta desde la mano hasta la base de este, obteniendo una señal en los sensores que dibuja una curva con forma de campana en la que se diferencian tres partes claras. La primera parte muestra un incremento pronunciado de los valores de la señal, indicando que un objeto penetra en el área de detección por uno de los flancos. Seguidamente, se obtiene un punto máximo de la curva. La presencia de varios puntos máximos indicaría que el objeto ha cambiado la trayectoria dentro del campo sensorizado. Finalmente, se obtiene en la curva una fase de bajada, simétrica a la de subida, dando por completada la detección del cubo y registrándolo para la puntuación del BBT. 


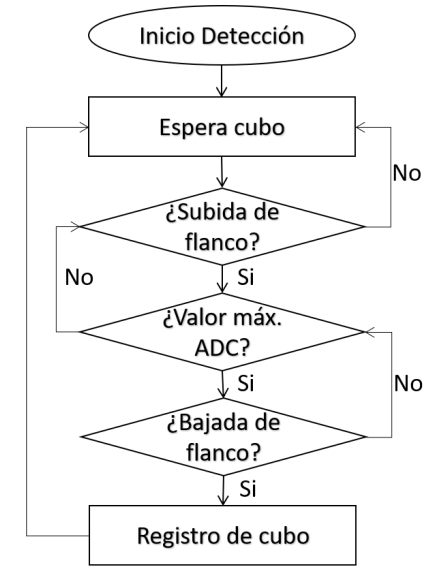

Figura 10: Diagrama general del proceso de detección de cubos en BBT.

\section{RESULTADOS}

En la Figura 11 se muestra el sistema implementado. La pieza divisoria central se ha replicado por medio de impresión 3D para alojar los sensores. Para la adquisición de datos e implementación del algoritmo se ha usado una placa Arduino Uno, conectando los sensores al bus I2C. Para cuantificar la efectividad del sistema de conteo implementado se han realizado varios experimentos en el laboratorio. En primer lugar, se busca cuantificar la tasa de acierto absoluta del sistema, desplazando cubos sin límite de tiempo hasta el total de 150 cubos. Por otro lado, se muestra la información adicional que se obtiene del desarrollo de la prueba, acotando el tiempo al definido por la normativa del test Finalmente, se ha buscado conocer la calidad de detección por zonas del compartimento.

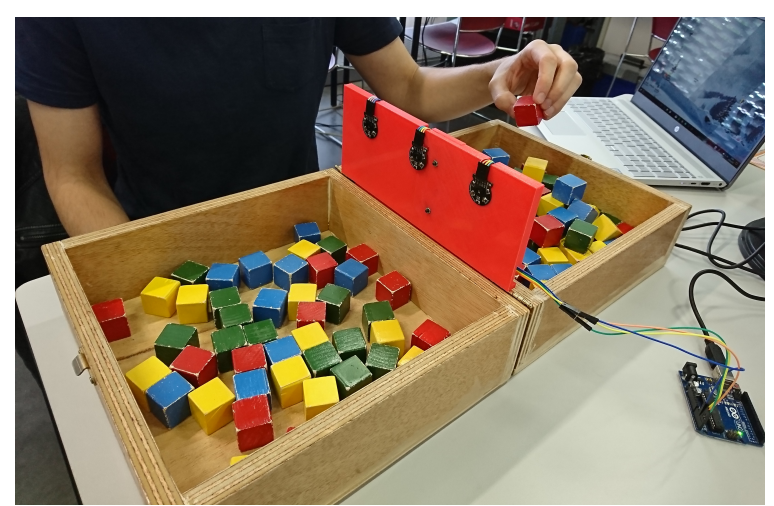

Figura 11: Prototipo del sistema implementado.

Los experimentos se dieron lugar en las instalaciones del Laboratorio de Robótica Asistencial en el Parque Científico Tecnológico de la Universidad Carlos III de Madrid (UC3M). Para ambos, se utilizó un sujeto sano sin problemas de movilidad en las extremidades superiores ni deterioro de destreza manual gruesa.
Tabla 1: Tasa de acierto en conteo automático a diferentes velocidades

\begin{tabular}{|c|c|c|c|c|c|}
\hline \multirow{2}{*}{$\begin{array}{l}\text { No. } \\
\text { cubos }\end{array}$} & \multicolumn{4}{|c|}{ Conteo automático } & \multirow{2}{*}{$\begin{array}{c}\text { Acierto } \\
(\%)\end{array}$} \\
\hline & $\mathrm{Vd} 1$ & $\mathrm{Vd} 2$ & $\mathrm{Vd} 3$ & $\mathrm{Vd} 4$ & \\
\hline 10 & 10 & 10 & 10 & 10 & 100,00 \\
\hline 20 & 20 & 20 & 20 & 20 & 100,00 \\
\hline 30 & 30 & 30 & 30 & 30 & 100,00 \\
\hline 40 & 40 & 40 & 38 & 38 & 97,50 \\
\hline 50 & 49 & 50 & 50 & 48 & 98,50 \\
\hline 60 & 60 & 58 & 59 & 55 & 96,67 \\
\hline 70 & 69 & 69 & 71 & 68 & 98,93 \\
\hline 80 & 79 & 79 & 80 & 76 & 98,13 \\
\hline 90 & 88 & 89 & 90 & 88 & 98,61 \\
\hline 100 & 97 & 95 & 98 & 90 & 95,00 \\
\hline 110 & 107 & 110 & 108 & 108 & 98,41 \\
\hline 120 & 120 & 122 & 120 & 114 & 99,17 \\
\hline 130 & 127 & 129 & 129 & 122 & 97,50 \\
\hline 140 & 141 & 136 & 140 & 134 & 98,39 \\
\hline 150 & 145 & 144 & 146 & 144 & 96,50 \\
\hline
\end{tabular}

\subsection{EFECTIVIDAD DEL SISTEMA}

Para determinar la efectividad absoluta en el conteo, se realizaron varias pruebas BBT sin límite de tiempo, desplazando cubos uno por uno en tandas de 10 cubos, hasta llegar a 150 cubos. Para este experimento se realizaron cuatro ensayos para cada tanda, variando la velocidad de desplazamiento (lento, media, rápida, aleatoria), mostrados en la Tabla 1. En total se realizaron 60 ensayos.

\subsubsection{Tasa de acierto en el conteo automático}

En la Figura 12 se presenta la tasa de acierto promedio en el conteo automático, mostrando un porcentaje de acierto constante elevado. En la detección de hasta 30 cubos se registran el $100 \%$ de desplazamientos, mientras que para puntuaciones mayores se obtiene una efectividad por encima del $97 \%$. Como resultado general se obtiene una tasa de acierto promedio de $98,22 \pm 1,41$.

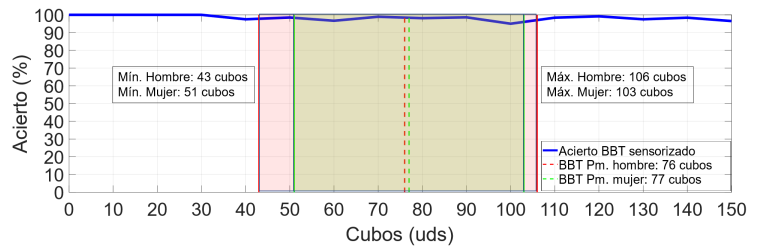

Figura 12: Tasa de acierto en la detección de cubos para BBT.

Comparando los resultados con los valores medios estándar [3], se observa que el sistema es capaz de operar en dicho rango de puntuaciones, contabilizando con éxito valores superiores a los habituales. 


\subsubsection{Datos adicionales obtenidos}

En la Figura 13 se muestran la distribución temporal de la detección de los cubos durante la realización de la prueba a diferentes velocidades de desplazamiento. El tiempo fue acotado a los 60 segundos definidos en la normativa del BBT.

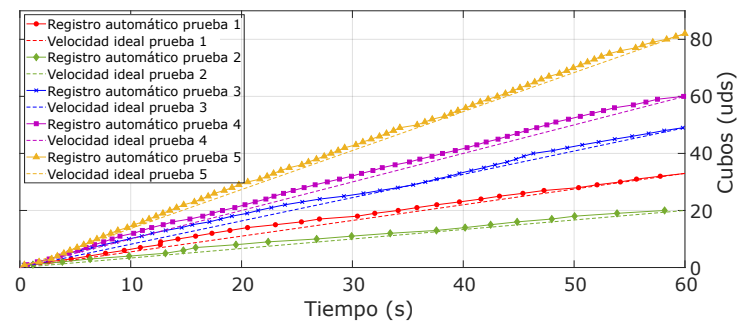

Figura 13: Resultados de conteo automático durante la prueba piloto BBT.

Se puede observar, que además la puntuación total obtenida de forma automática, se proporciona al terapeuta de información adicional como la frecuencia de desplazamiento de cubos, nivel de dispersión o tiempos parciales. Además, puede conocer con exactitud los puntos de la prueba en los que un paciente ha presentado problemas o dificultades para mantener un ritmo constante. Esta información adicional, que no se puede obtener con el test tradicional, puede permitir al terapeuta hacer una valoración más amplia de la condición del paciente.

\subsubsection{Limitaciones}

Para determinar las limitaciones en la detección de acuerdo a la zona del compartimento, se dividió el área de recepción de cubos en trece zonas (Figura 14-a). Se realizaron diez ensayos divididos en grupos de dos, en los que se dejaron caer cubos desde una altura de $20 \mathrm{~cm}$, en series de 10 cubos.

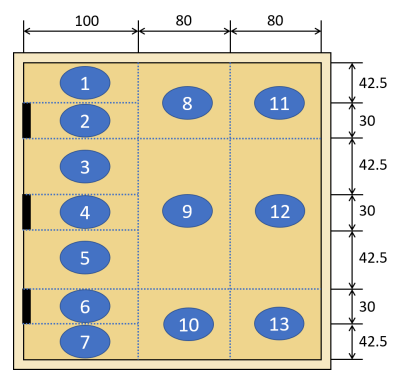

(a)

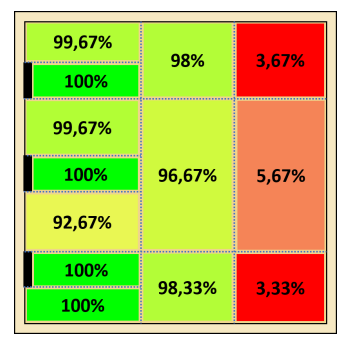

(b)
Figura 14: Porcentaje de detección en el interior de un compartimento. (a) Zonas de detección, (b) Tasa de acierto según la zona del compartimento.

Los resultados se muestran en la Figura 14-b. Se observa una tasa de acierto superior al $98 \%$ en las zonas cercanas a los sensores y superiores al $96 \%$ en la franja central del compartimento. Además, en las zonas frontales a los sensores se obtienen un $100 \%$ de acierto. Por el contrario, en el lateral y esquinas mas alejadas no se produce detección, obteniendo valores cercanos al $4 \%$. Como se ha comentado anteriormente, este error es asumible teniendo en cuenta la dinámica del desplazamiento de cubos en el BBT.

\section{CONCLUSIONES}

En este artículo, se ha presentado el desarrollo de un sistema basado en sensores de proximidad para la automatización de resultados del test de destreza y coordinación manual BBT. Se ha comprobado que la sensorización de la pieza divisora es adecuada, puesto que no altera o entorpece la ejecución de la prueba.

Por un lado, la tasa de acierto conseguida hasta los 150 cubos es elevada en todos los casos, con un porcentaje promedio de $98,22 \pm 1,41$. Se ha comprobado que los cambios de luz no afectan, siendo el sistema lo suficientemente estable como para operar tanto con luz natural y como artificial. Sin embargo, la tasa de acierto varía ligeramente en función de la velocidad de desplazamiento de los cubos, disminuyendo para velocidades altas. Así, los fallos de detección pueden provenir de las áreas con baja o nula sensorización, ya que los cubos pueden ser lanzados a una mayor distancia de la barrera central. Para solventar estos inconvenientes se puede utilizar sensores de mayor rango de alcance.

Por otro lado, considerando las normas del BBT, la media de cubos desplazados por personas jóvenes es de $77 \pm 31$ cubos, lo que refuerza la viabilidad del método propuesto ya que la tasa de acierto es buena hasta los 150 cubos.

Como trabajos futuros se valora la combinación de este sistema basado en sensores junto con otro basado en visión por computador [5] para mejorar aún más el registro de datos y adquisición de información adicional. El método combinado permitiría evitar detección de falsos positivos como el desplazamiento de la mano sin cubo. También, se espera realizar ensayos con usuarios reales.

\section{Agradecimientos}

La investigación que lleva a estos resultados ha recibido financiación de los proyectos ROBOHEALTH-A (DPI2013-47944-C4-1-R) y ROBOESPAS (DPI2017-87562-C2-1-R), financiados por el Ministerio de Economía y Competitividad español, y del proyecto RoboCity2030-III-CM (S2013/MIT-2748), financiado por Programas de 
Actividades de I+D en la Comunidad de Madrid y co-financiado por Fondos Estructurales de la UE.

\section{English summary}

\section{Automatic cube counting for manual dexterity assessment using proximity sensors}

\begin{abstract}
In this article, the development of a system to automatically obtain the score of the Box and Blocks Test (BBT), a manual dexterity assessment method, using proximity sensors is presented. The BBT's assessment is based on the maximum number of cubes that a person is able to displace during a time window. Thus, the movement trajectory of the cubes have been sensorized using infrared-based proximity sensors. First, the methodology used for automation of the test scoring is presented, including the sensors'description and the prototype design. Then, the obtained success rate in cube counting is shown. Finally, the conclusions and future works are exposed. The results support the use of automated methods in rehabilitation processes, providing more objective results and additional information about the user performance.
\end{abstract}

Keywords: Assessment, Automatic, Upper extremity, Manual dexterity, Rehabilitation, Proximity sensors.

\section{Referencias}

[1] C.-P. Hsiao, C. Zhao, and E. Y.-L. Do. The digital box and block test automating traditional post-stroke rehabilitation assessment. In Pervasive Computing and Communications Workshops (PERCOM Workshops), 2013 IEEE International Conference on, pages 360-363. IEEE, 2013.

[2] T. Lee, K. Leo, E. Chew, L. Zhao, and S. Sanei. Automated scoring of rehabilitative tests via time-frequency informed singular spectrum analysis. In Computational Intelligence for Multimedia Understanding (IWCIM), 2015 International Workshop on, pages 1-5. IEEE, 2015.
[3] V. Mathiowetz, G. Volland, N. Kashman, and K. Weber. Adult norms for the box and block test of manual dexterity. American Journal of Occupational Therapy, 39(6):386-391, 1985.

[4] Modern Device. Si1143 proximity sensors. URL https://moderndevice.com/ product/si1143-proximity-sensors/. [Accedido 30 May 2018].

[5] E. D. Oña, A. Jardón, and C. Balaguer. The automated box and blocks test an autonomous assessment method of gross manual dexterity in stroke rehabilitation. In Y. Gao, S. Fallah, Y. Jin, and C. Lekakou, editors, Towards Autonomous Robotic Systems, pages 101-114, Cham, 2017. Springer.

[6] E. D. Oña, R. Cano-de la Cuerda, P. SánchezHerrera, C. Balaguer, and A. Jardón. A review of robotics in neurorehabilitation: Towards an automated process for upper limb. Journal of healthcare engineering, 2018 (9758939):1-19, 2018

[7] P. Otten, J. Kim, and S. H. Son. A framework to automate assessment of upper-limb motor function impairment: A feasibility study. Sensors, 15(8):20097, 2015.

[8] J. J. M. F. van der Putten, J. C. Hobart, J. A. Freeman, and A. J. Thompson. Measuring change in disability after inpatient rehabilitation: comparison of the responsiveness of the barthel index and the functional independence measure. Journal of Neurology, Neurosurgery 8 Psychiatry, 66(4):480-484, 1999.

[9] E. Wade, A. R. Parnandi, and M. J. Matarić. Automated administration of the wolf motor function test for post-stroke assessment. In Pervasive Computing Technologies for Healthcare (PervasiveHealth), 2010 4th International Conference on-NO PERMISSIONS, pages 1-7. IEEE, 2010.

[10] J. Wang, L. Yu, J. Wang, L. Guo, X. Gu, and Q. Fang. Automated fugl-meyer assessment using svr model. In Bioelectronics and Bioinformatics (ISBB), 2014 IEEE International Symposium on, pages 1-4. IEEE, 2014.

[11] World Health Organization. Global diffusion of ehealth: Making universal health coverage achievable, 2016. URL http://www . who.int/goe/publications/ global_diffusion/en/. [Accedido 30 May 2018].

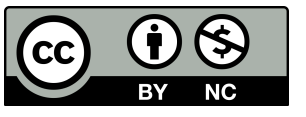

(C) 2018 by the authors. Submitted for possible open access publication under the terms and conditions of the Creative Commons Attribution CC-BY-NC 3.0 license (http://creativecommons.org/licenses/by-nc/3.0/). 